\title{
Estado actual del cultivo de mandioca en la República Argentina.
}

\author{
Angela María Burgos* \\ * Facultad de Ciencias Agrarias-Universidad Nacional del Nordeste (UNNE) \\ e-mail: burgosangela@agr.unne.edu.ar
}

Conferencia dictada en el II Congreso Latinoamericano y Caribeño de Mandioca y XVII Congreso

Brasilero de Mandioca. Belem, Brasil 12-16 de marzo de 2018.

Argentina es el más austral entre los países productores de mandioca de Latinoamérica, donde se la cultiva desde la época anterior a la colonización europea. Actualmente, el cultivo se realiza en la zona nordeste (NEA) integrada por las provincias de Corrientes, Chaco, Formosa y Misiones, donde es reconocido como un cultivo regional, de importancia cultural y socioeconómica.

Las áreas de cultivo responden a un clima mayormente subtropical húmedo que se clasifica como Cfa por el sistema Köppen-Geiger, con una frecuencia de ocurrencia de heladas de $0,5 \%$ y con una frecuencia media anual de 11,5 días con heladas agrometeorológicas $\left(\leq 3^{\circ} \mathrm{C}\right)$ (Murphy, 2008). Estos factores climáticos condicionan muchas prácticas de cultivo y obligan a cortar y conservar el material de multiplicación (tallos) durante los casi cuatro meses que se extiende el invierno. La plantación se realiza a partir de la segunda quincena de agosto, en el periodo libre de heladas y el ciclo productivo se prolonga hasta fines del otoño.

\section{Relevamiento de datos y estadísticas de producción.}

La revisión bibliográfica de referencia nos lleva en primera instancia a los datos de superficie cultivada con mandioca en Argentina, provista por un organismo internacional FAOSTAT (2017), que se muestran en la Tabla 1.

Tabla 1. Producción de mandioca en Argentina durante el período 2010-2016.

\begin{tabular}{cccc}
\hline Año & $\begin{array}{c}\text { Superficie } \\
\text { (ha) }\end{array}$ & $\begin{array}{c}\text { Rendimiento } \\
\text { (kg ha-1) }\end{array}$ & $\begin{array}{c}\text { Producción } \\
\text { (Toneladas) }\end{array}$ \\
\hline 2010 & 18.000 & 10.000 & 181.800 \\
2011 & 18.500 & 10.000 & 185.000 \\
2012 & 18.700 & 10.000 & 187.000 \\
2013 & 18.500 & 9.945 & 184.000 \\
2014 & 18.642 & 9.980 & 186.057 \\
2015 & 18.998 & 10.014 & 190.255 \\
2016 & 19.095 & 10.012 & 191.193 \\
\hline Promedio & 18.633 & 9.993 & 186.472 \\
\hline
\end{tabular}

Fuente FAOSTAT, 2017
La contribución de Argentina a la producción mundial con un promedio de 186.500 toneladas de mandioca (Tabla 1), no resultaría significativa en este contexto y la ubicaría por detrás del puesto 40 en volumen de producción mundial.

A nivel país, se cuenta con los relevamientos realizados a través del Censo Nacional Agropecuario del año 2002 (CNA-2002) y 2008 (CNA-2008), en este último año el prestigio del Indec (Instituto Nacional de Estadística y Censo) comenzaba a ser cuestionado y finalmente se cuenta con los datos provistos por los Ministerios de Producción de cada Provincia (Tabla 2). Es importante destacar que una proporción de la producción de este cultivo no se registra en las estadísticas oficiales de manera precisa y confiable, en coincidencia con la escasez de recursos destinados para un cultivo considerado parte de una "economía regional", que se desarrolla en tierras consideradas marginales (fuera de la región pampeana) por lo que ver su evolución en el tiempo resulta difícil. Esta carencia de estadísticas de producción, industrialización y comercialización representa un cuello de botella para la toma de decisiones de los actores del sector, así como para el diseño de estrategias sectoriales.

La Tabla 2 muestra la "lamentable" situación de contar con tan solo 15.000 ha de mandioca en el país. El adjetivo "lamentable" responde a la información publicada por el Instituto Nacional de Tecnología Agropecuaria (INTA) en Bongiovanni et al. (2012) donde reportaban 40.000 ha de mandioca en el año 2009 y donde se exponía la expectativa de llegar a 45.000 ha en el 2016 y a 50.000 ha en el 2020, incrementando la producción de 200.000750 .000 toneladas de raíces. Esas proyecciones se enmarcaron dentro del PEA 2010-2020 (Plan Estratégico Agrolimentario y Agroindustrial Participativo y Federal) que proponía firmemente un cambio en el modelo de desarrollo del sector Agroalimentario y Agroindustrial y postulaba un salto paradigmático desde la Argentina "granero del mundo", a un país proveedor de productos elaborados de alto valor y alta calidad; claramente esto no se ha reflejado y menos aún, en el cultivo de mandioca. 
Tabla 2. Superficies cultivadas con mandioca en hectáreas (ha) en las provincias productoras de Argentina.

\begin{tabular}{lccccc}
\hline & \multicolumn{5}{c}{ Superficies cultivadas con mandioca en hectáreas (ha) } \\
Fuente de Información & Misiones & Corrientes & Formosa & Chaco & Total \\
\hline CNA-2002 (1/7/01-30/6/02) & 9.097 & 1.642 & 1.527 & 924 & 13.190 \\
CNA-2008 (1/7/07-1/5/08) & 6700 & 61 & 148 & 77 & 6.886 \\
Ministerios de Producción & 10.000 & 3.533 & 1153 & 450 & 15.131 \\
Provinciales & $(2016)$ & (prom 2008-2015) & (prom 2012 2017) & (2016) & \\
\hline
\end{tabular}

Referencias de tabla: CNA: Censo Nacional Agropecuario.

Tabla 3. Producción total promedio $(\mathrm{t}$ año-1 $)$ y rendimientos promedios $\left(\mathrm{t} \mathrm{ha} \mathrm{a}^{-1}\right)$ de mandioca en las provincias productoras de Argentina.

\begin{tabular}{lccccc}
\hline \multirow{2}{*}{$\begin{array}{l}\text { Fuente de } \\
\text { Información }\end{array}$} & \multicolumn{5}{c}{ Producción Total Promedio $\left(\mathrm{t}\right.$ año $0^{-1}$ ) y Rendimiento Promedio $\left(\mathrm{t} \mathrm{ha}{ }^{-1}\right)$} \\
\cline { 2 - 6 } & Misiones & Corrientes & Formosa & Chaco & Total \\
\hline Ministerios de & 165.000 & 42.500 & 11.530 & 4.500 & 223.530 \\
Producción & 16,5 & 12,07 & 10 & 10 & 12,14 \\
\hline
\end{tabular}

Para ser optimistas, lo "bueno" es ver que a pesar de la constricción de la superficie plantada, el aumento de los rendimientos promedio permitió mantener estable la producción en $223.500 \mathrm{t}$.

(Tabla 3). El rendimiento promedio nacional de $12,14 \mathrm{t} \mathrm{ha}^{-1}$ de raíces, se halla muy por encima de las 9,9 $\mathrm{t} \mathrm{ha}^{-1}$ estimadas por FAOSTAT (2017) para la producción argentina (Tabla 1) y no tan lejano al promedio mundial de $12,84 \mathrm{t} \mathrm{ha}^{-1} \mathrm{y}$ de $12,88 \mathrm{t} \mathrm{ha}^{-1}$ para el continente americano citados por Howeler (2014). Sin embargo, aún distan demasiado, del de nuestros vecinos Brasil y Paraguay, y de los rendimientos potenciales alcanzables con las condiciones agroecológicas regionales del NEA.

Al procesar los datos más actuales provistos por los Ministerios Provinciales de la distribución porcentual de la producción argentina de mandioca (Figura 1), se visualiza el protagonismo de la Prov. de Misiones con $73,82 \%$ de la producción total, seguida por Corrientes $(19,01 \%)$, Formosa $(5,15 \%)$ y Chaco $(2,01 \%)$.

Los agricultores argentinos que cultivan mandioca son pequeños productores de escasos recursos que trabajan parcelas que no suelen superar una hectárea, aplican tecnología tradicional y muy básica. Resultan también escasos los programas estatales de incentivo y desarrollo de la actividad "mandioquera" en la región, pese a tener un gran potencial agroindustrial.

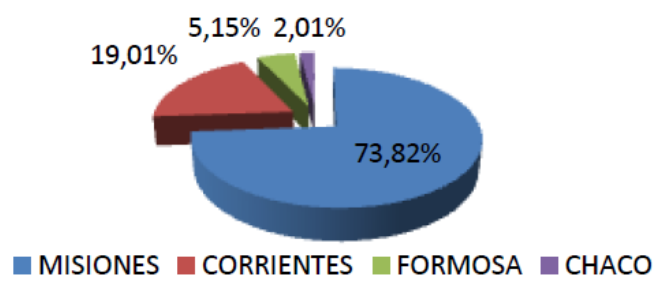

Figura 1. Distribución porcentual de la producción de mandioca en las provincias argentinas.
El único cambio fuerte y destacable se originó principalmente en Misiones, ya que la provincia incrementó su volumen de producción aún a expensas de la fuerte disminución del área plantada. La provincia de Misiones posee cuatro cuencas productoras de mandioca (Aristizabal y Calle, 2015) y según datos de la Subsecretaría de Producción Vegetal de esta Provincia, cerca de 12.000 pequeños productores.

Otro dato destacable es que la provincia de Misiones, es la única que cuenta hoy con un "Cluster de Mandioca" que fue organizado por el Ministerio del Agro y la Producción de la provincia a través de la Subsecretaría de la Industria y que se ha puesto en marcha en el año 2014. Es importante resaltar que el "Cluster" explica el 100\% de la producción industrial de Argentina y es el centro de la actividad en nuestro país tanto de la producción primaria como de la industrial, que se abastece únicamente con la producción propia de esa provincia. Dicho Cluster está integrado por numerosos organismos estatales y privados, entre ellos el Ministerio del Agro y la Producción, el Instituto Nacional de Tecnología Agropecuaria (INTA), el Instituto Nacional de Tecnología Industrial (INTI), las Universidades de Misiones (UNAM) y la de Buenos Aires (UBA), la Unidad para el Cambio Rural (UCAR) dependiente del Ministerio de Agroindustria de la Nación, productores primarios e industriales. El Banco Interamericano de Desarrollo (BID) otorgó casi 4,2 millones pesos desde 2015 para el desarrollo de seis proyectos que han buscado mejorar la competitividad de los productores de mandioca en Misiones, y que en parte lo están logrando. Las otras tres provincias apenas cuentan con algunas Cooperativas de productores que entre otras actividades cultivan pequeñas superficies de mandioca, y actualmente no cuentan con ninguna planta industrial funcionando.

\section{Aprovechamiento del cultivo en Argentina.}

El sector mandioquero presenta dos actividades desarrolladas que únicamente utilizan las raíces: 
i) La primaria, representada por la producción de raíces para consumo fresco como hortícola lleva casi el $82 \%$ de toda la producción y logra satisfacer la demanda nacional. Gran parte de la producción hortícola de mandioca en el país, se destina al autoconsumo y el resto se comercializa a granel en mercados locales. Casi la totalidad de las producciones de Corrientes, Formosa y Chaco y el $75 \%$ de la de Misiones se destina a la producción de raíces en fresco y no se industrializa. En Argentina, los mayores esfuerzos se focalizan en satisfacer el autoconsumo de raíces; después en abastecer el mercado del producto en fresco y por último en proveer a la industria local. De hecho, aún en Misiones (centro de la actividad industrial) el $50 \%$ de la producción de raíces se destina al autoconsumo, y de la otra mitad, $25 \%$ se destina a la comercialización en fresco y solo el restante $25 \%$ se industrializa.

El consumo estuvo siempre muy arraigado en la dieta de los colonos del noreste de nuestro país, pero en las dos últimas décadas el producto en fresco pasó a ser requerido en distintos mercados, dado que el hábito de su consumo se extendió progresivamente a otras ciudades. Actualmente la mandioca está presente en bocas de expendio mayoristas y minoristas de localidades de Buenos Aires, Santa Fe y Córdoba, situación que se tradujo en un interesante movimiento económico para las diferentes localidades productoras. Muchos son llevados a mercado sin ningún valor agregado, pero en los hipermercados también se las comercializa parafinadas, envasadas al vacío, refrigeradas, congeladas y supercongeladas.

Solo clones dulces $(<50 \mathrm{mg} / \mathrm{kg}$ de cianuro en las raíces frescas) se cultivan en Argentina. Si se trata de variedades tempranas, la recolección de raíces destinadas al consumo en fresco comienza a partir del octavo mes de plantación. Las variedades tardías se cosechan después del décimo mes. El INTA reconoce en Misiones ocho cultivares para consumo humano, dos de los cuales coinciden con las variedades indígenas (Pomberí, Coloradita, Concepción, Rocha, Papa blanca, Pombero guazú, Amarilla y Corrientes 74).Las agencias de desarrollo para pequeños productores promocionan variedades para consumo provenientes del banco de germoplasma del INTA El Colorado (provincia de Formosa) que agregan a las anteriores los cvs. Caoba, Blanca Caá Guazú, Cerro Azul 25/1 y Verde Olivo, entre otros.

ii) La secundaria, abarca la industrialización de raíces cuyo único derivado explotado es la obtención de harina y fundamentalmente fécula (almidón nativo). Para poder satisfacer las demandas de industrias diversas, algunas cooperativas están comenzando a producir almidones modificados, generando más valor agregado en origen. Empresas radicadas en las Provincias de Formosa y Corrientes dejaron de operar debido a la insuficiente cantidad de raíces. Actualmente en Corrientes solo se elabora fécula artesanal que se comercializa en ferias francas locales para uso doméstico en la elaboración del "chipá", semejante a su "pão de queijo".

Las fábricas de almidón que son un importante eslabón de la cadena de valor de la mandioca, se abastecen comprando las raíces a socios de las cooperativas o a productores independientes de la cuenca productora misionera. Desde el punto de vista comercial, la capacidad industrial instalada permite procesar unas 292.000 toneladas de raíces al año, equivalentes a unas 73.000 toneladas de fécula. A pesar de existir actualmente funcionando unas 11 plantas industriales procesadoras de fécula de mandioca en Misiones (4 cooperativas y 7 empresas familiares), no pueden alcanzar a cubrir su capacidad productiva por falta de materia prima, generándose una competencia entre sí por acceder al escaso producto, que además no posee la calidad necesaria. Los ocho cultivares utilizados con destino industrial son los mismos destinados al consumo humano (exceptuando Amarilla y Rocha) a los que se le agregan Blanca de Santa Catarina y CA 25-1. La diversidad que exhibe no representa un atributo valorado y al mismo tiempo no constituye una materia prima estandarizada.

A partir de los 8 y hasta los 18 meses de plantación se cosechan las raíces para la industria y la molienda de mandioca se realiza en el período que va de marzo a septiembre, que es el período en el cual se dispone de materia prima.

La capacidad ociosa supera el $40 \%$, generando un alto grado de subocupación en la industria. La realidad es que por falta de materia prima solamente se muelen entre 42.000 y 50.000 toneladas, equivalentes a una producción anual de fécula de 10.300 a 12.500 toneladas considerando una eficiencia de extracción de almidón de mediana a alta de $25 \%$. De esta manera, la producción resulta insuficiente para abastecer la demanda nacional de 15.000-18.000 toneladas de fécula, por lo que entre el 57 y en el mejor de los casos el $80 \%$ se puede llegar a cubrir con la producción nacional. Argentina importa un promedio de 5.100 $\mathrm{t}$ año ${ }^{-1}$ de fécula (Figura 2), provenientes principalmente de Tailandia, Paraguay y Brasil; siendo este un tema muy complejo en su administración para la cadena (CAFAGDA, 2017).

Lo destacable es el dato de que la fécula de mandioca representó el 35\% del total de los almidones importados durante el período 2000-2016 (Figura 3) lo que pone de manifiesto la importancia del mismo para la industria nacional como fortaleza y oportunidad (CAFAGDA, 2017).

Las principales demandantes de fécula son las industrias alimenticias, químicas, farmacéuticas, adhesivas y textiles, concentradas mayoritariamente en 


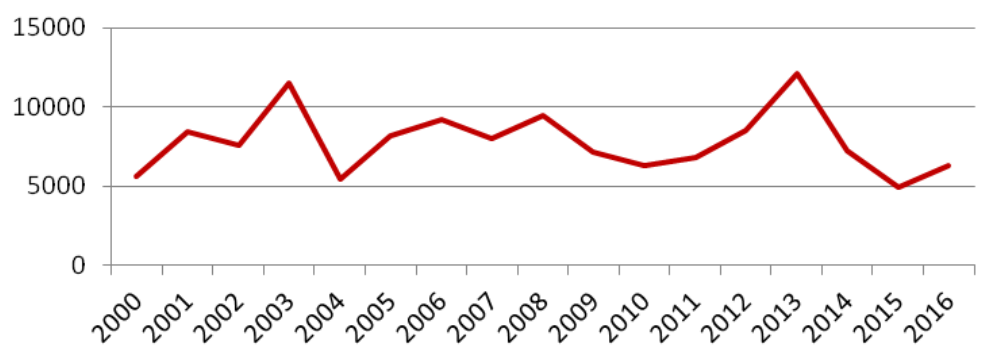

Figura 2. Importaciones de mandioca o fécula de mandioca en toneladas en el período 2000-2016

(Fuente CAFAGDA, 2017).

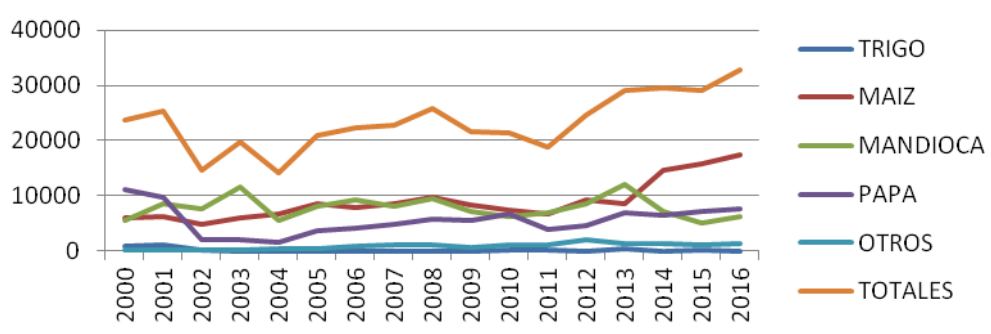

Figura 3. Importaciones de almidones en toneladas en el período 2000-2016.

Fuente CAFAGDA, 2017

las provincias de Buenos Aires, Santa Fe, Córdoba y Mendoza. De las industrias alimenticias, las de chacinados son las más grandes demandantes $(90 \%$ del total) de fécula de mandioca.

Actualmente la industria farmacéutica aumentó su demanda de almidones simples, ésta es satisfecha por la producción nacional en bajo porcentaje y de almidones modificados provistos por importaciones de industrias principalmente brasileras. Se observa un continuo crecimiento en el consumo de productos y manufacturas que insumen almidón modificado en sus procesos productivos, pero de las 11 industrias que funcionan en Misiones, solo tres cuentan con instalaciones adecuadas para elaborar almidones modificados. De esta manera, uno de los retos del sector industrial misionero consiste en la elaboración de almidones modificados adaptando su infraestructura.

Últimamente la fécula de mandioca también es muy requerida por industrias relacionadas a la elaboración de diversos productos para celíacos que según la propia Asociación Celíaca Argentina, afecta al 1\% de la población y alrededor de 400.000 argentinos y sus respectivas familias son potenciales consumidores de productos libres de gluten.

En Argentina la fécula se envasa en bolsas de $1 \mathrm{~kg}$ para su comercialización en supermercados y dietéticas y, llega a la industria panificadora y frigorífica en envases de 10 y $25 \mathrm{~kg}$. La harina suele comercializarse en comercios dietéticos como complemento de fibra, para acompañar las comidas o para mezclar con leche complementando algunos cereales. El mercado nacional de demanda se proyecta creciente.

\section{Consideraciones finales}

Se detecta claramente que el principal cuello de botella para la captura de oportunidades es que no hay suficiente cantidad de materia prima para abastecer a la industria. Para alcanzarlo: i) los productores deben tomar conciencia de que necesitan reconvertirse y tecnificar su cultivo. ii) Los técnicos deben reforzar las prácticas de manejo con paquetes tecnológicos apropiados para cada provincia en particular. Resta además promover el aprovechamiento integral del cultivo para la alimentación animal en un país ganadero (follaje, descartes) que ya se está difundiendo desde las Facultades de Agronomía y Veterinaria de la UNNE. iii) La industria debe invertir y adaptarse a los requerimientos del mercado, agregando valor en origen como almidones modificados, bioplásticos y biocombustibles, entre otros. iv) A la comunidad científica nos queda la obligación de responder a las necesidades de la sociedad. Es menester investigar para aumentar la productividad a través de la mejora en el uso de insumos, de recursos, con sistemas sostenibles propios para la región, garantizando la seguridad alimentaria. La biotecnología es muy prometedora respecto al aumento de la productividad y la conservación de los recursos naturales. v) Por último, la posibilidad de continuar las investigaciones en este cultivo en particular, ha sido, es y será responsabilidad del Estado y de las instituciones de nuestro país. Quienes conformamos parte de las Universidades y sus institutos dependientes; del INTA, del INTI, y del CONICET (Consejo Nacional de Investigaciones Científicas y Técnicas), estamos trabajando para ello. Finalmente, es competencia del Estado incentivar la creación de nuevos polos industriales en las otras tres provincias, sustentadas en políticas definidas de desarrollo. La actividad ha venido mostrando importantes mejoras, pero todavía no hay un total aprovechamiento de la red de recursos que la integran.

Como corolario, considero que el cultivo de mandioca en el NEA en el largo plazo puede estar condicionado a sucumbir como tal o a subsistir, pero para ello deberá transformarse para un mundo que exige un producto distinto y con la calidad adecuada y certificada para un determinado demandante, que reúna criterios sustentables en términos de buenas prácticas agrícolas y de manufactura. Si en Argentina se considerase a la mandioca como un producto estratégico para el desarrollo de numerosas industrias y se le otorgara el tratamiento correspondiente en términos 
de inversiones, muy certeramente podría darse un vuelco en el desarrollo del sector agroalimentario e industrial regional, contribuyendo a la generación de riqueza y de empleo rural y urbano, con el valioso sello del valor agregado en origen de productos diversificados, originales e innovadores.

\section{Referencias bibliográficas}

Asociación Celíaca Argentina. En: http://www.celiaco.org. ar/ [fecha de consulta: enero 2018].

Aristizabal, J \& Calle, F. Producción, Procesamiento, Usos y Comercialización de Mandioca. Cuaderno Tecnológico $\mathrm{N}^{\mathrm{o}} 22$, p. 41, 2015.

Bongiovanni, R, MorandI, J., Troilo, L. (Eds). Competitividad y calidad de los cultivos industriales: caña de azúcar, mandioca, maní, tabaco, té y yerba. INTA EEA Manfredi, 205 p., 2012.
CAFAGDA. Cámara Argentina de Fabricantes de Almidones, Glucosas, Derivados y Afines. http://www.cafagda.com.ar/estad_impo.htm. [fecha de consulta: enero 2018].

CNA 2002. En: https://www.indec.gob.ar/index_agropecuario.asp. [fecha de consulta: enero 2018].

CNA 2008 En: https://www.indec.gov.ar/ [fecha de consulta: enero 2018].

FAOSTAT (División Estadística de la Food and Agriculture Organization of the United Nations) En: http://www.fao. org/faostat/es/\#home. 2017. [fecha de consulta: enero 2018].

Howeler, R. Sustainable soil and crop management of cassava in Asia. CIAT. Cali, Colombia, p. 280, 2014.

Murphy, G.M. (Ed.). Atlas Agroclimático de la Argentina. Facultad de Agronomía, Universidad de Buenos Aires. Argentina. p. 130,2008. 